\title{
Integrin $\alpha_{\mathrm{v}} \beta_{3}$-targeted gold nanoshells augment tumor vasculature-specific imaging and therapy
}

This article was published in the following Dove Press journal:

International Journal of Nanomedicine

26 January 2011

Number of times this article has been viewed

\author{
Huan Xie' \\ Parmeswaran Diagaradjane ${ }^{2}$ \\ Amit A Deorukhkar ${ }^{2}$ \\ Beth Goins ${ }^{3}$ \\ Ande $\mathrm{Bao}^{3}$ \\ William T Phillips ${ }^{3}$ \\ Zheng Wang ${ }^{4}$ \\ Jon Schwartz ${ }^{5}$ \\ Sunil Krishnan ${ }^{2}$ \\ 'Department of Pharmaceutical \\ Sciences, College of Pharmacy and \\ Health Sciences, Texas Southern \\ University, Houston, TX, USA; \\ ${ }^{2}$ Department of Radiation Oncology, \\ Division of Radiation Oncology, the \\ University of Texas MD Anderson \\ Cancer Center, Houston, TX, USA; \\ ${ }^{3}$ Department of Radiology, the \\ University of Texas Health Science \\ Center at San Antonio (UTHSC- \\ San Antonio), San Antonio, TX, USA; \\ ${ }^{4}$ MPI Research, Inc., Mattawan, MI, \\ USA; ${ }^{5}$ Nanospectra Biosciences, Inc., \\ Houston, TX, USA
}

Correspondence: Sunil Krishnan Department of Radiation Oncology, Unit 097, The University of Texas MD Anderson Cancer Center, I5I 5 Holcombe Blvd, Houston, TX 77030, USA

$\mathrm{Tel}+\mathrm{I} 7135632377$

Fax + I 7|35632366

Email skrishnan@mdanderson.org
Purpose: Gold nanoshells (NSs) have already shown great promise as photothermal actuators for cancer therapy. Integrin $\alpha_{v} \beta_{3}$ is a marker that is specifically and preferentially overexpressed on multiple tumor types and on angiogenic tumor neovasculature. Active targeting of NSs to integrin $\alpha_{v} \beta_{3}$ offers the potential to increase accumulation preferentially in tumors and thereby enhance therapy efficacy.

Methods: Enzyme-linked immunosorbent assay (ELISA) and cell binding assay were used to study the in vitro binding affinities of the targeted nanoconjugate NS-RGDfK. In vivo biodistribution and tumor specificity were analyzed using ${ }^{64} \mathrm{Cu}$-radiolabeled untargeted and targeted NSs in live nude rats bearing head and neck squamous cell carcinoma (HNSCC) xenografts. The potential thermal therapy applications of NS-RGDfK were evaluated by subablative thermal therapy of tumor xenografts using untargeted and targeted NSs.

Results: ELISA and cell binding assay confirmed the binding affinity of NS-RGDfK to integrin $\alpha_{\mathrm{v}} \beta_{3}$. Positron emission tomography/computed tomography imaging suggested that tumor targeting is improved by conjugation of NSs to cyclo(RGDfK) and peaks at $\sim 20$ hours postinjection. In the subablative thermal therapy study, greater biological effectiveness of targeted NSs was implied by the greater degree of tumor necrosis.

Conclusion: The results presented in this paper set the stage for the advancement of integrin $\alpha_{\mathrm{v}} \beta_{3}$-targeted NSs as therapeutic nanoconstructs for effective cancer therapy.

Keywords: nanoparticle, cyclo(RGDfK), cancer, thermal ablation

\section{Introduction}

Gold nanoshells (NSs) are core/shell particles comprising a gold shell and a dielectric silica core with peak plasmon resonances tunable to desired wavelengths by adjusting the relative core and shell thicknesses. At near infrared (NIR) wavelengths, light penetrates deep within the tissue (up to several centimeters), making this an optimal wavelength for biomedical applications. Indeed, NSs that absorb maximally in the NIR wavelength and efficiently convert incident light to heat can be readily synthesized (eg, a 120-nm core diameter and a 14-nm-thick shell result in an absorption peak between $780 \mathrm{~nm}$ and $800 \mathrm{~nm}$ ). Biodistribution studies indicate that untargeted NSs passively accumulate in solid tumors through the enhanced permeability and retention (EPR) effect, do not enter healthy tissues to the same extent, and appear to be safe and well tolerated. ${ }^{1,2}$ This tumor-specific accumulation and NIR activation have been exploited for thermal ablation of solid tumors using NIR illumination. 3,4 Studies have suggested that gold NSs are suitable candidates for clinical use in thermal therapy applications in cancer. To further enhance tumor selectivity, active targeting 
strategies have been pursued by many investigators. ${ }^{5,6}$ Given the relatively large size of $\mathrm{NSs}^{7}{ }^{7}$ they are unlikely to penetrate deep into tumor parenchyma but are readily sequestered within the perivascular space. Therefore, active targeting to antigens specific to tumor cells is not expected to achieve substantially higher tumor penetration.

In contrast to tumor antigen-targeting strategies, tumor vascular targeting has some unique advantages. Integrins are a family of cell adhesion molecules consisting of two noncovalently bound transmembrane subunits ( $\alpha$ and $\beta$ ) that form heterodimers. ${ }^{8}$ The integrin $\alpha_{v} \beta_{3}$ binds to arginine-glycine-aspartic acid (RGD)-containing components of the extracellular matrix, blood, and cell surface proteins. Multiple lines of evidence suggest that this integrin heterodimer can serve as a target for tumor neovascular imaging and image-guided cancer therapies. First, integrin $\alpha_{v} \beta_{3}$ is significantly upregulated on endothelium during angiogenesis and on fast-growing solid tumor cells but not on quiescent endothelium and normal tissues. ${ }^{9-12}$ Second, RGD molecular probes have been developed for imaging integrin expression using different modalities, such as magnetic resonance imaging, ${ }^{13}$ ultrasound, ${ }^{14,15}$ optical imaging, ${ }^{16-19}$ positron emission tomography (PET), ${ }^{20-22}$ and single-photon emission computed tomography (SPECT). ${ }^{22,23}$ Third, reagents that bind selectively to integrin $\alpha_{\mathrm{v}} \beta_{3}$ can be designed by cyclizing peptides with selected sequences around the RGD and by synthesizing RGD mimics. ${ }^{8}$ Fourth, in many cancers, higher numbers of tumor-associated vessels express integrin $\alpha_{\mathrm{v}} \beta_{3}$ than vessels in normal tissue. ${ }^{24}$ Lastly, inhibition of integrin $\alpha_{v} \beta_{3}$ using monoclonal antibodies, cyclic RGD peptide antagonists (Figure 1A), and peptidomimetics has been shown to induce endothelial cell apoptosis, inhibit angiogenesis, and increase endothelial monolayer permeability. ${ }^{25,26}$ Collectively, these studies suggest that integrin $\alpha_{\mathrm{v}} \beta_{3}$ can serve as a selective, but not specific, target for imaging and therapy of cancer. Further, cyclic RGD peptides can serve as conduits to anchor probes on these integrins, and when combined with the EPR effect mediated by leaky vasculature and insufficient lymphatic drainage of tumors, additional accumulations of these conjugated probes may enhance this selectivity. In addition to RGD peptides, some research groups have synthesized small molecule antagonists based on RGD motifs to achieve comparable affinity with integrin $\alpha_{\mathrm{v}} \beta_{3} \cdot{ }^{27-29}$

In this study we compared the binding affinities to integrin $\alpha_{\mathrm{v}} \beta_{3}$ of the cyclo(RGDfK) (cyclo(Arg-Gly-Asp-D-Phe-Lys)) peptide and the synthesized antagonist 4-[2-(3,4,5,6-tetrahydropyrimidin-2-ylamino)-ethyloxy]benzoyl-2-(S)-[N-(3-aminoneopenta-1-carbamyl)]-aminoethylsulfonylamino-h-alanine hydrochloride (IAC) (Figure 1B) ${ }^{27}$ and their NS conjugates by three different (ELISAs). The binding specificity of NSRGDfK to integrin $\alpha_{\mathrm{v}} \beta_{3}$-expressing U87 tumor cells was then validated by silver staining assays. In vivo biodistribution and tumor specificity were analyzed using ${ }^{64} \mathrm{Cu}$-radiolabeled untargeted NSs and targeted NSs in live rats bearing head and neck squamous cell carcinoma (HNSCC) xenografts. Lastly, the relative ability of untargeted and targeted NSs to induce tissue damage after induction of subablative temperatures was assessed in a tumor xenograft model.

\section{Material and methods NS synthesis and conjugation}

Gold-silica NSs were synthesized as previously described. ${ }^{30}$ NS formation was assessed using an ultraviolet-visible (UVVIS) spectrophotometer (U-0080D, Hitachi) and Zetasizer (Nano-ZS, Malvern Instruments, Malvern, UK). Integrin $\alpha_{\mathrm{v}} \beta_{3}$-targeting peptide cyclo-(RGDfK) (molecular weight $[\mathrm{MW}]=617.71)$ and control peptide cyclo-(Arg-Ala-AspD-Phe-Lys) (cyclo-(RADfK), MW = 603.68) were purchased from Peptides International (Louisville, KY, USA). Integrin $\alpha_{\mathrm{v}} \beta_{3}$ antagonist IAC (MW $\left.=622.14\right)$ was provided

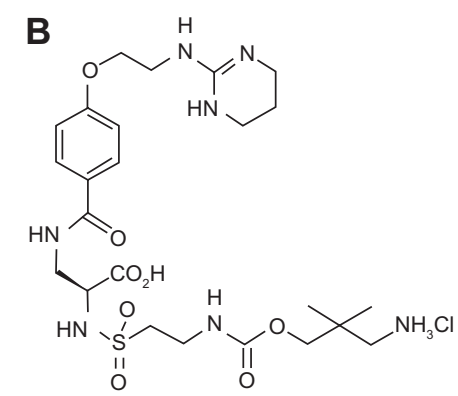

Figure I Structures of cyclo (Arg-Gly-Asp-D-Phe-Lys) (RGDfK) (A) and the antagonist, 4-[2-(3,4,5,6-tetrahydro-pyrimidin-2-ylamino)-ethyloxy]benzoyl-2-(S)-[N-(3-aminoneopenta-I-carbamyl)]-aminoethylsulfonylamino-h-alanine hydrochloride (IAC) (B). 
by Dr Narasimhan Danthi from the Molecular Imaging Laboratory at National Institutes of Health. Bifunctional ortho-pyridyldisulfide-polyethylene glycol 2000-N-hydroxysuccinimide ester (OPSS-PEG2k-NHS) was purchased from Nektar (Huntsville, AL, USA). Chelating agent S-2-(4aminobenzyl)-1,4,7,10-tetraazacyclododecane tetraacetic acid (DOTA) was purchased from Macrocyclics (Dallas, TX, USA). The free amine groups of RGDfK, RADfK, IAC, and DOTA were conjugated to OPSS-PEG-NHS by mixing 1:1 molar ratios overnight at $\mathrm{pH} 7.4$ at room temperature (RT). The resulting OPSS-PEG conjugates were then mixed with NS solution (in $10 \mathrm{mM}$ phosphate buffer, $\mathrm{pH}$ ) at 10,000:1 molar ratio overnight at RT on a shaker, allowing the OPSS group to conjugate to the gold surface of the particles. The mixture was centrifuged, and the supernatant with unconjugated OPSSPEG was removed. The pellets of NS-peptide, NS-IAC, or NS-DOTA were resuspended in phosphate buffer and analyzed by a spectrophotometer and Zetasizer to determine the NS concentration and size, respectively, for further conjugation. To prepare NS with both RGDfK and DOTA on the surface, OPSS-PEG-RGDfK and OPSS-PEG-DOTA were mixed with NS at a 5000:1 molar ratio followed by the incubation and separation steps.

\section{In vitro binding affinity assays Competitive ELISA for RGDfK and IAC}

Purified integrin $\alpha_{v} \beta_{3}$ protein (Chemicon International, Temecula, CA, USA) was coated on 96-well plates at $0.1 \mu \mathrm{g} /$ well. After overnight incubation at $4^{\circ} \mathrm{C}$, the plates were washed (washing buffer: $1 \mathrm{X}$ phosphate buffered saline [PBS] with $0.05 \%$ Tween 20) and then blocked with blocking buffer (1X PBS with $2 \%$ bovine serum albumin [BSA]) at RT for 2 hours. The blocking buffer was removed, and the plates were inoculated in triplicate with both RGDfK and IAC at a typical starting concentration of $0.125 \mu \mathrm{g} / \mathrm{mL}$. Serial dilutions were prepared in the 96-well plates with blocking buffer supplemented with $0.9 \mathrm{mM}$ of $\mathrm{Ca}^{2+}$ and $0.5 \mathrm{mM}$ of $\mathrm{Mg}^{2+}$. After 30 minutes of incubation, $0.2 \mu \mathrm{g}$ of biotinylated vitronectin solution (Molecular Innovations, MI, USA) was added to each well as a standard competitor. The plates were incubated at RT for 3 hours then washed, and the bound vitronectin was detected using $0.01 \mu \mathrm{g} /$ well of avidin-horse radish peroxidase (HRP) conjugate (Pierce, Rockford, IL, USA). After washing, $100 \mu \mathrm{L}$ of substrate solution was added to each well and then the luminescence was read (Figure 2A). The resulting luminescence signal intensity was plotted against RGDfK and IAC concentration and fitted by SigmaPlot 10.0 (SYSTAT Software, Inc., San Jose,
CA, USA), and the concentrations of inhibitor producing $50 \%$ inhibition $\left(\mathrm{IC}_{50}\right)$ of vitronectin binding to integrin $\alpha_{\mathrm{v}} \beta_{3}$ were calculated based on the curve fittings.

\section{Sandwich ELISA for NS-RGDfK and NS-IAC}

Purified integrin $\alpha_{\mathrm{v}} \beta_{3}$ protein was coated on 96-well plates as described previously. The plates were inoculated in triplicate with NS-PEG5K (control), NS-RGDfK, and NS-IAC, with a typical starting NS concentration of 1 optical density (OD). 1 OD NS is approximately 3 4 pM. Serial dilutions were prepared in the 96-well plates using blocking buffer containing $\mathrm{Ca}^{2+}$ and $\mathrm{Mg}^{2+}$ and then incubated at RT for 1 hour. The detection antibody biotin-anti-PEG (Epitomics, Burlingame, CA, USA), which recognizes the methoxy group of the PEG5K, was added to each well at $0.05 \mu \mathrm{g} /$ well. The plates were incubated at RT for 1 hour then washed, and the bound biotin-anti-PEG was detected using avidin-HRP conjugate at $0.005 \mu \mathrm{g} /$ well. After washing, $100 \mu \mathrm{L}$ of substrate solution was added to each well and then the luminescence was read (Figure 2B). The resulting luminescence signal intensity was plotted against NS concentration and fitted by SigmaPlot 10.0. The half-maximal binding values $(\mathrm{Kd})$ were calculated based on the curve fittings.

\section{Competitive ELISA for NS-RGDfK and NS-IAC}

Purified integrin $\alpha_{\mathrm{v}} \beta_{3}$ protein was coated on 96-well plates as described previously. The plates were inoculated in triplicate with RADfK (control), RGDfK, and IAC with a typical starting concentration of $50 \mu \mathrm{M}(100 \mu \mathrm{L} /$ well $)$. Serial dilutions were prepared in the 96-well plates using blocking buffer containing $\mathrm{Ca}^{2+}$ and $\mathrm{Mg}^{2+}$ and then incubated at RT for 0.5 hour, followed by addition of NS-RGDfK ( $0.5 \mathrm{OD}, 100 \mu \mathrm{L} /$ well) as competitor, and incubation for 2.5 hours. After extensive washing, the detection antibody biotin-anti-PEG $(0.05 \mu \mathrm{g} /$ well $)$ was added to each well. The plates were incubated at RT for 1 hour then washed, and the bound biotin-anti-PEG was detected using avidin-HRP conjugate at $0.005 \mu \mathrm{g} /$ well with 1-hour incubation. After washing, $100 \mu \mathrm{L}$ of substrate solution was added to each well and then the luminescence was read (Figure 2C). The resulting luminescence signal intensity was plotted against NS concentration and fitted by SigmaPlot 10.0, and the $\mathrm{IC}_{50}$ values were calculated based on the curve fittings.

\section{Cell adhesion assay}

U87 human glioblastoma cell lines (ATCC, Manassas, VA, USA) were cultured under standard conditions and grown to $80 \%$ confluence in 48 -well plates. Cells were rinsed three times in PBS (pH 7.4). NS-RGDfK and NS-PEG5K 

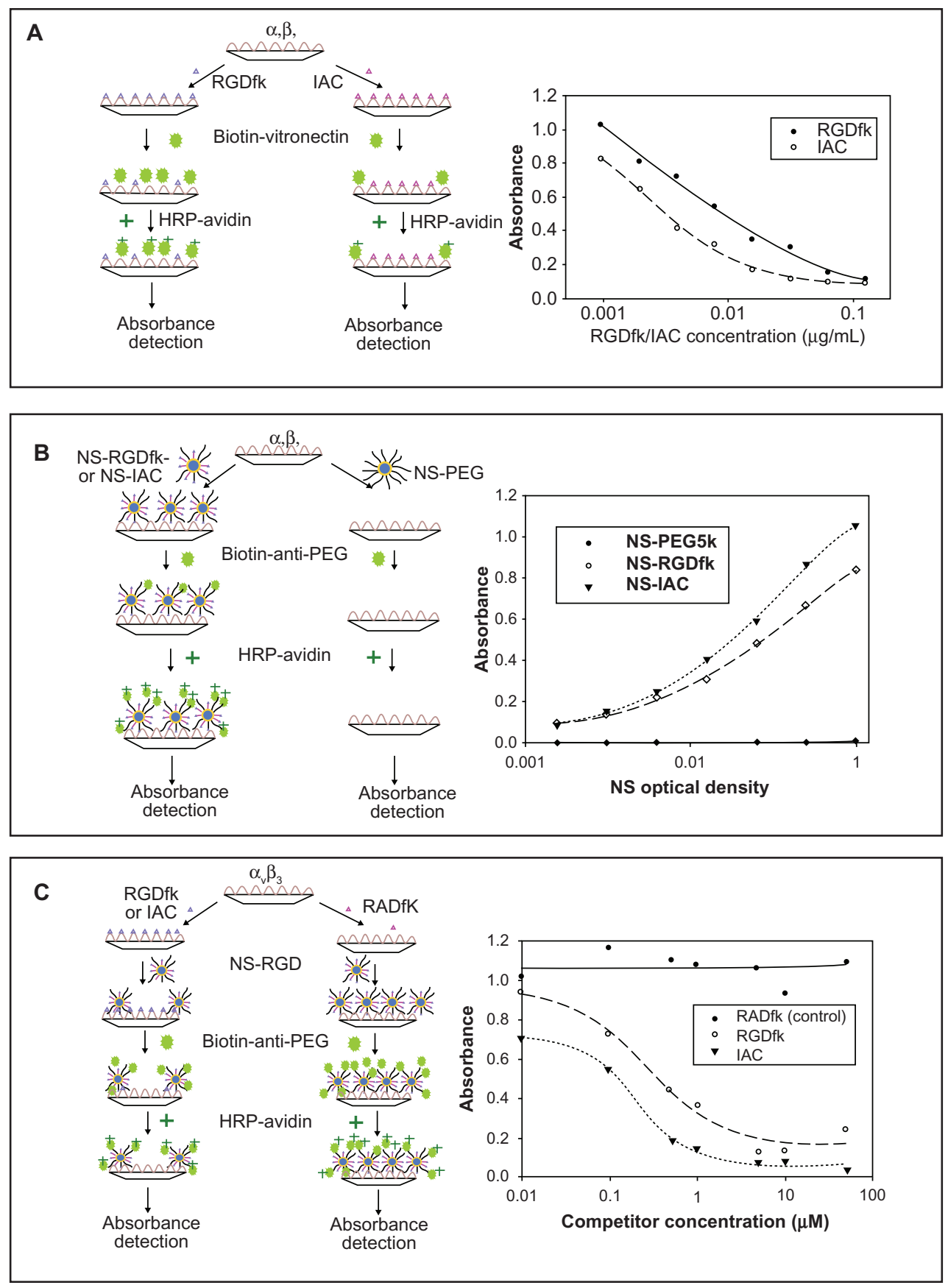

Figure 2 Schematic drawings (left) and results (right) of the three ELISAs. (a) Competitive ELISA of vitronectin against RGDfK and the antagonist IAC on an integrin $\alpha_{v} \beta_{3}$ coated plate. The absorbance of HRP-avidin is proportional to the amount of the biotinylated vitronectin on the plate. (b) Sandwich ELISA of NS-PEG5K (control), NS-RGDfK and NSIAC on an integrin $\alpha_{v} \beta_{3}$ coated plates. The absorbance of HRP-avidin is proportional to the amount of the blocker PEG5K on NS and therefore, to the number of NS binding to the integrin $\alpha_{v} \beta_{3}$. (c) Competitive ELISA of NS-RGDfK against RGDfK and IAC on an integrin $\alpha_{v} \beta_{3}$ coated plates. The absorbance of HRP-avidin is proportional to the amount of the blocker PEG5K on NS and therefore, to the number of NS binding to the integrin $\alpha_{v} \beta_{3}$. All values are means of duplicate assays in the three ELISA experiments.

Abbreviations: ELISA, enzyme-linked immunosorbent assay; HRP, horse radish peroxidase; IAC, 4-[2-(3,4,5,6-tetrahydro-pyrimidin-2-ylamino)-ethyloxy]benzoyl-2-(S)[N-(3-amino-neopenta-I-carbamyl)]-aminoethylsulfonylamino-h-alanine hydrochloride; NS, nanoshell; PEG, polyethylene glycol; RADfK, Arg-Ala-Asp-D-Phe-Lys; RGDfK, Arg-Gly-Asp-D-Phe-Lys.

were mixed in the cell growth medium to obtain samples with 1.25 OD, which were then added to the U87-coated plate with $300 \mu \mathrm{L} /$ well and incubated for 45 minutes at $37^{\circ} \mathrm{C}$. Cells in PBS served as negative control. Cells were rinsed three times in PBS and fixed with 2\% glutaraldehyde for 15 minutes and then rinsed three times with deionized water. A silver staining kit (Amersham Biosciences, Piscataway, NJ, USA) was used to stain cells, depositing silver on the NSs surfaces, to allow visualization of NS binding to cells via light microscopy with $40 \times$ magnification. 


\section{Radiolabeling and imaging}

Radiolabeling procedure

All the radioisotope work was performed at the Department of Radiology, University of Texas Health Science Center (UTHSC)-San Antonio, TX, USA. ${ }^{64} \mathrm{CuCl}_{2}$ (Washington University, St Louis, MO, USA) was diluted in $30 \mathrm{mM}$ ammonia citrate buffer (pH 6.5). Then, $\sim 1000 \mu \mathrm{Ci}$ of ${ }^{64} \mathrm{Cu}$ was added to $200 \mu \mathrm{L}$ of the solutions of NS-DOTA and NS with both RGDfK and DOTA (concentrations of NSs were about $1 \mathrm{nM}$ ) and incubated at $37^{\circ} \mathrm{C}$ for 90 minutes. Then the blocking agent PEG5K-SH (Nektar) was added to NS solution at 300,000:1 molar ratio and incubated at RT on a shaker for 1 hour. The mixtures were then centrifuged to remove unconjugated ${ }^{64} \mathrm{Cu}$ and PEG5K. The ${ }^{64} \mathrm{Cu}$ activity of the supernatant and pellet was measured in a dose calibrator (Atomlab 100, Biodex, Shirley, NY, USA). Labeling efficiencies were calculated by using the equation [activity in pellet/ (activity in supernatant + activity in pellet) $\times 100$.

\section{Animal model and in vivo PET imaging}

An HNSCC xenograft model in nude rats was established via subcutaneous inoculation of the HNSCC cell line SCC-4. ${ }^{31}$ Animal experiments with radioactive agents were carried out at UTHSC according to the NIH Animal Use Guidelines. On the day of imaging, the average tumor volume of the nude rats was $1.2 \mathrm{~cm}^{3}$. During all procedures, the nude rats $(\sim 150 \mathrm{~g})$ were anesthetized with intraperitoneal Avertin ${ }^{\circledR}(400 \mathrm{mg} / \mathrm{kg})$ by intraperitoneal injection. ${ }^{64} \mathrm{Cu}-\mathrm{NS}\left(\sim 800 \mu \mathrm{Ci}\right.$ of $\left.{ }^{64} \mathrm{Cu}\right)$ and ${ }^{64} \mathrm{Cu}-\mathrm{NS}-\mathrm{RGDfK}\left(\sim 600 \mu \mathrm{Ci}\right.$ of $\left.{ }^{64} \mathrm{Cu}\right)$ were injected manually into the rats' tail veins at about $0.5 \mathrm{~mL}$ per minute. PET imaging was performed at 1 hour, 4 hours, 20 hours, and 44 hours postinjection of radiolabeled NS with the FLEX X-PET/CT/SPECT scanner (Gamma Medica-Ideas, Inc., Northridge, CA, USA) followed by CT image acquisition ( $80 \mathrm{kVp}, 0.25 \mathrm{~mA}, 256$ projections).

\section{In vivo analysis of thermoablation efficacy}

In a subcutaneous colorectal cancer xenograft model using HCT116 human tumor cells in nude mice, $100 \mu \mathrm{L}(\sim 0.4 \mathrm{nM})$ of NS-PEG5K and NS-RGDfK solutions were injected via the tail vein of the animals (two mice in each group). After 24 hours, thermocouple probes were inserted into the center and the base of the tumor at about $5 \mathrm{~mm}$ depth from the surface. T-type (copper-constantan) needle thermocouple probes (HYP1-30-1/2-T-G-60-SMP-M, Omega Engineering) were used. The probe alone did not generate heat as tested by irradiating the probe with the laser. The NIR laser power was set at values predicted to result in subablative temperatures based on an Arrhenius model $1^{32}$ of thermal injury $\left(47^{\circ} \mathrm{C}\right.$ for 10 minutes was predicted to achieve $50 \%$ cell kill with untargeted NSs, a level that would allow comparison with agents that potentially increased the efficacy of thermoablation). With a $1.2 \mathrm{~W} 75 \%$ duty cycle, $808 \mathrm{~nm}$ NIR laser and a spot size of $1 \mathrm{~cm}$, consistent temperature elevation to $47^{\circ} \mathrm{C}$ was achieved within 5 minutes at the center of the tumor and was maintained steadily for 10 minutes in the untargeted NS group. Tumors were excised 2 hours after thermal therapy following pretreatment with NS-PEG5K and conjugated NS-RGDfK and stained with hematoxylin and eosin. Tissues were observed by Olympus DP 70 camera fitted on a Nikon Microphot-FXA microscope, and images captured using Olympus DP software.

\section{Results and discussion}

NSs used in this study were manufactured to comprise a silica core $(\sim 120 \mathrm{~nm}$ in diameter) and a gold shell $(8 \sim 10 \mathrm{~nm})$ to absorb light at NIR wavelengths. The UV-VIS spectrum confirmed the NS absorbance peak at $\sim 760 \mathrm{~nm}$. Zetasizer measurements confirmed the NS diameter of $140 \mathrm{~nm}$ and zeta potential of $-50 \mathrm{mV}$. By adapting a method that has been developed for conjugation of molecules with amine groups, ${ }^{2,4}$ NSs were conjugated to cyclic RGD peptides, IAC, or DOTA through bifunctional PEG. Dynamic lightscattering measurements showed that after surface modification the NS size increased to $\sim 170 \mathrm{~nm}$ in diameter, and zeta potential changed to around $-5 \mathrm{mV}$.

The binding affinities and specificities of RGDfK and IAC for integrin $\alpha_{\mathrm{v}} \beta_{3}$ receptor were measured by a competitive ELISA. Serial dilutions of these peptides were assayed for their ability to compete with biotinylated vitronectin for the immobilized integrin $\alpha_{v} \beta_{3}$ receptor. After obtaining the curve fittings by SigmaPlot (Figure 2A), the calculated $\mathrm{IC}_{50}$ concentration of inhibitors RGDfK and IAC for vitronectin binding to integrin $\alpha_{v} \beta_{3}$ were $4.05 \mathrm{nM}$ and $3.38 \mathrm{nM}$, respectively. These results confirmed the relatively comparable binding affinities of IAC and RGDfK to integrin $\alpha_{v} \beta_{3}$ protein and closely matched the reported $\mathrm{IC}_{50}$ of $2.94 \pm 0.19 \mathrm{nM}$ for IAC by Burnett et al. ${ }^{27}$ The lower $\mathrm{IC}_{50}$ of RGDfK than that noted by Burnett et al for cyclo-(RGDfV) $(6.41 \pm 0.49 \mathrm{nM})$ might be due to the different amino acids in the two peptides valine $(\mathrm{V})$ and lysine $(\mathrm{K})$, with the amine group in lysine potentially affording greater protein binding.

Subsequently, a sandwich ELISA was used to compare the binding affinities of NS-RGDfK, NS-IAC, and NS-PEG5K (control, no targeting molecule). Serial dilutions of these agents were added to an integrin $\alpha_{v} \beta_{3}$-coated plate prior to washing 
and detection of bound NSs. As NSs are not readily detected on ELISA plates, the PEG coating was used as a surrogate for their presence. This sandwich ELISA therefore employed a biotinylated anti-PEG antibody that recognizes the methoxy group of the PEG5K. The antibody was subsequently detected by HRP-avidin. Figure 2B shows that control NS-PEG5K had no detectable binding to the integrin $\alpha_{\mathrm{v}} \beta_{3}$, whereas NS-RGDfK and NS-IAC had increasing binding to the integrin $\alpha_{\mathrm{v}} \beta_{3}$ with increasing concentration. The Kd values for NS-RGDfK and NS-IAC were 0.267 OD and 0.288 OD, respectively, calculated based on the SigmaPlot curve fitting.

In the third competitive ELISA, serial dilutions of the competitors RGDfK, IAC, and RADfK (negative control) were pre-incubated in separate wells of an integrin $\alpha_{\mathrm{v}} \beta_{3}$ coated plate. The targeted NS-RGDfK (concentrated to give $0.5 \mathrm{OD}$ at $800 \mathrm{~nm}$ ) particles were then added to all the wells to compete with RGDfK or IAC for binding. Biotin-labeled anti-PEG antibody and HRP-avidin was used for detection as before. Figure $2 \mathrm{C}$ shows that negative control RADfK did not have any detectable competition with NS-RGDfK, whereas RGDfK and IAC did compete with NS-RGDfK for binding to the integrin. The $\mathrm{IC}_{50} \mathrm{~s}$ of RGDfK and IAC were 0.267 and $0.197 \mu \mathrm{M}$, respectively. Because of the relatively small difference in binding affinity between RGDfK and IAC for all three ELISAs, further in vitro and in vivo experiments were performed with NS-RGDfK.

The in vitro binding affinity and specificity of NS-RGDfK was tested using U87 glioblastoma cells that express abundant integrin $\alpha_{\mathrm{v}} \beta_{3}$. Cells were incubated with PBS, NS-PEG5K, and NS-RGDfK and treated with a silver staining kit. Figure 3 shows that the two controls (PBS and NS-PEG5K) had no detectable binding to cells, and NS-RGDfK bound specifically and avidly to integrin $\alpha_{v} \beta_{3}$-overexpressing cells. For all the in vitro affinity studies, we found that a small amount of $\mathrm{Ca}^{2+}$ $(0.9 \mathrm{mM})$ and $\mathrm{Mg}^{2+}(0.5 \mathrm{mM})$ must be added to the buffer or cell growth medium for RGDfK to bind to the integrin $\alpha_{\mathrm{v}} \beta_{3}$. Hu et al have reported how $\mathrm{Ca}^{2}$ and $\mathrm{Mg}^{2+}$ regulate ligand binding to $\beta_{3}$ integrins. ${ }^{33}$ They concluded that low concentrations of $\mathrm{Ca}^{2+}(\mu \mathrm{M})$ promoted the binding of the ligand, whereas higher concentrations of $\mathrm{Ca}^{2+}(\mathrm{mM})$ blocked the binding. This study revealed that there are two classes of cation binding sites on the $\beta_{3}$-containing integrins. The first class of sites promotes ligand binding to integrin when occupied by divalent cations, and the second class of sites is allosteric to the ligand binding site and highly specific for $\mathrm{Ca}^{2+}$ and when occupied increases the dissociation rate between RGD and integrin. ${ }^{34}$

After confirming biochemical and in vitro binding affinities of NS-RGDfK, we evaluated its stability, biodistribution, and efficacy in imaging integrin $\alpha_{\mathrm{v}} \beta_{3}$ in a solid tumor model in vivo by dual labeling NSs with a radionuclide $\left({ }^{64} \mathrm{Cu}\right)$ and the RGDfK peptide. We have used both ${ }^{64} \mathrm{Cu}$ and ${ }^{111} \mathrm{In}$ for radiolabeling of gold NSs. ${ }^{35,36}$ The PET and SPECT images of the rats showed that both ${ }^{64} \mathrm{Cu}$ and ${ }^{111} \mathrm{In}$ appeared to be useful for tracking the in vivo distribution of NSs. The biodistribution data determined with ${ }^{111} \mathrm{In}$ were very close to those of ${ }^{64} \mathrm{Cu}$, but image quality was better with the ${ }^{64} \mathrm{Cu}$. This is likely due to the more efficient detection of the ${ }^{64} \mathrm{Cu}$ with the PET ring coincidence imaging system than with the SPECT rotating

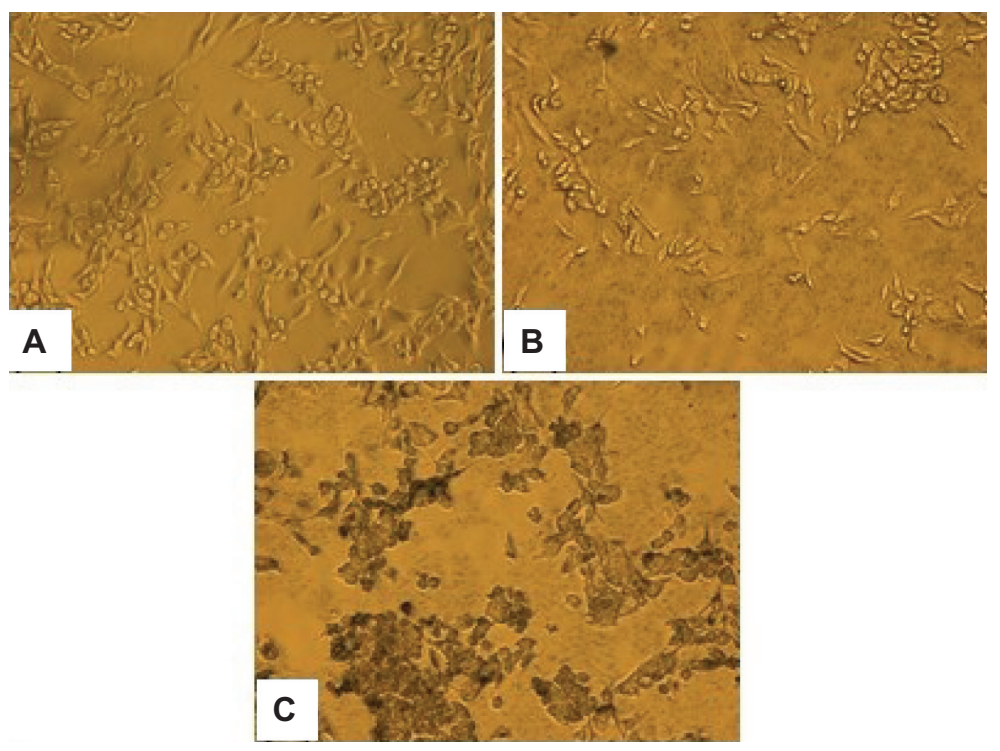

Figure 3 Silver staining of integrin $\alpha_{v} \beta_{3}$ overexpressing U87 glioma cancer cells with PBS (A), NS-PEG5K (B), and NS-RGDfK (C) solutions. Images were taken by light microscope with 40× magnification.

Abbreviations: NS, nanoshell; PBS, phosphate buffered saline; PEG, polyethylene glycol; RGDfK, Arg-Gly-Asp-D-Phe-Lys. 
camera system. Therefore, PET imaging with ${ }^{64} \mathrm{Cu}$ was used for the NS imaging studies described in this paper.

The ${ }^{64} \mathrm{Cu}$ radiolabeling efficiencies were $81.3 \%$ and $84.2 \%$ for NS and NS-RGDfK, respectively. Using PET/CT imaging, we monitored the in vivo distribution of radiolabeled NSPEG and NS-RGDfK at various time points after intravenous injection through the tail vein into nude rats with HNSCC xenografts. Figure 4 shows PET/CT fusion images (top) and PET sagittal images (bottom) of rats at 1 hour, 4 hours, 20 hours, and 44 hours postinjection. Our previous studies suggested that the radioisotope was not cleaved from the NSs, so we believe that our images and biodistribution data truly reflect the location of NSs. ${ }^{2}$ Neither NS-PEG nor NS-RGDfK showed obvious accumulation within tumors at 1 hour postinjection. However, NS-RGDfKs progressively accumulated in the tumor, beginning at 4 hours postinjection and reaching an apparent maximum accumulation at approximatly 20 hours postinjection, followed by gradual decline in tumor-specific accumulation by 44 hours postinjection, whereas untargeted NS accumulation in tumors was less pronounced during each of these time points. These results suggest that RGDfK conjugation improves NS accumulation within tumors and that laser treatment for photothermal applications should optimally occur at $\sim 20-44$ hours postinjection. The animals were sacrificed at 46 hours postinjection, and major organs were collected for analysis of specific tissue accumulations. The amount of NSs
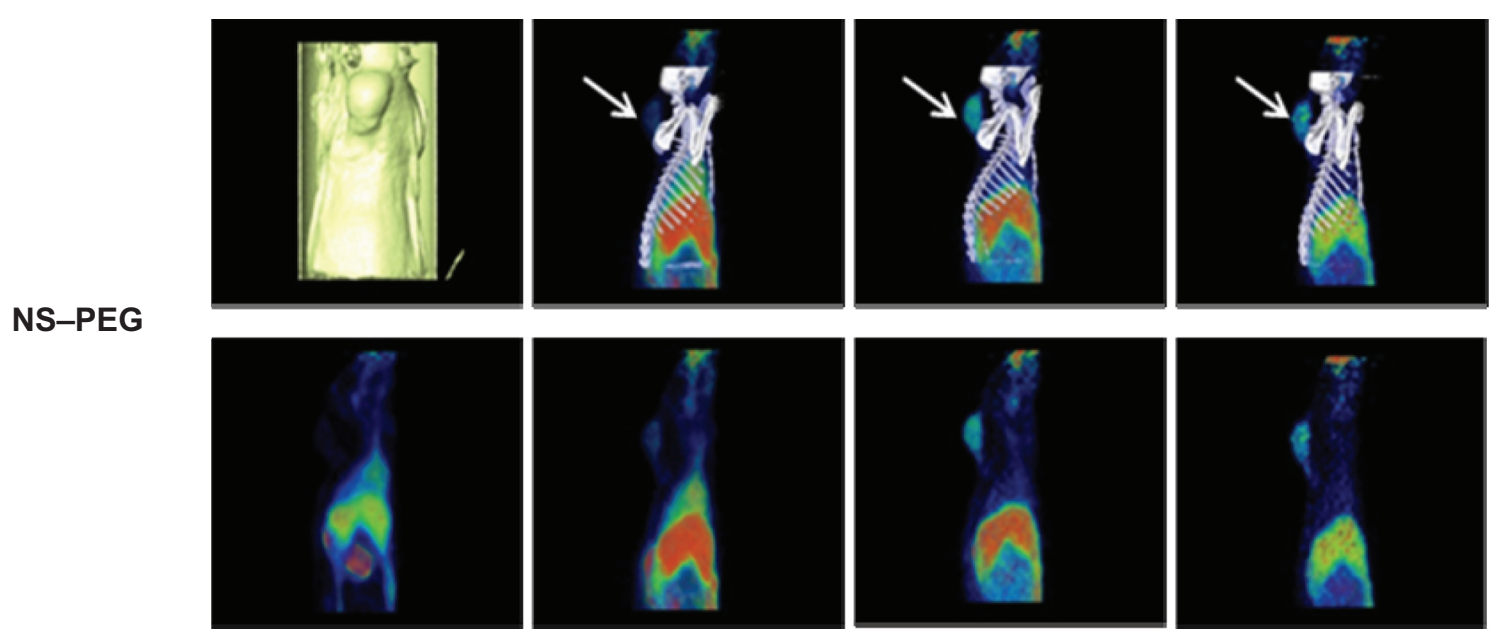

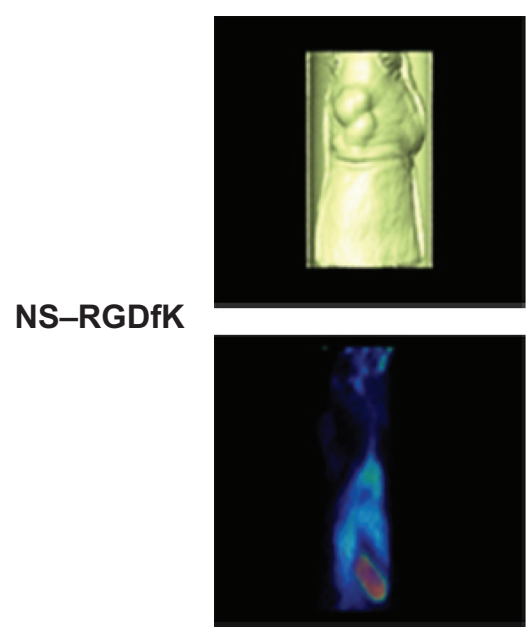

1 hour
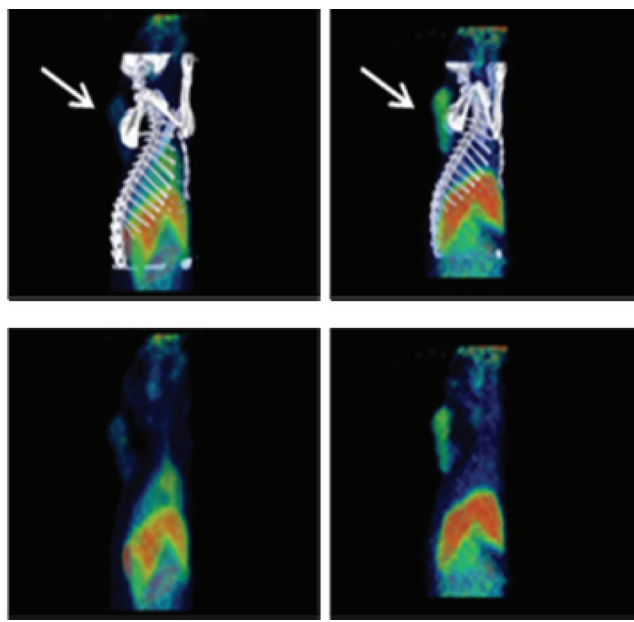

4 hours

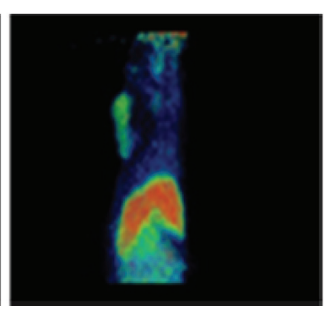

20 hours
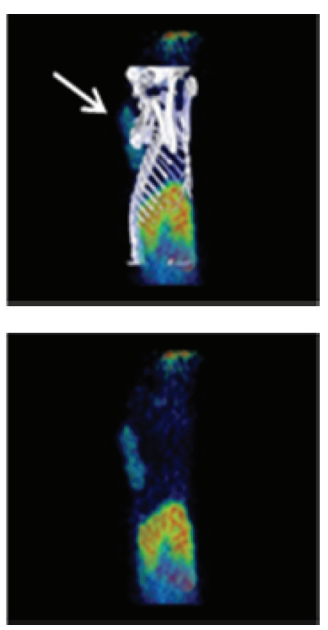

44 hours

Figure 4 PET/CT fusion and PET sagittal images of two nude rats bearing HNSCC xenograft at I hour, 4 hours, 20 hours, and 44 hours post tail-vein injection of ${ }^{64}$ Cu-NS and ${ }^{64} \mathrm{Cu}-\mathrm{NS}-\mathrm{RGDFK}$. The arrows are pointing at the tumor.

Abbreviations: CT, computed tomography; HNSCC, head and neck squamous cell carcinoma; NS, nanoshell; PEG, polyethylene glycol; PET, positron emission tomography; RGDfK, Arg-Gly-Asp-D-Phe-Lys. 
in the tissue samples as estimated by gamma well counter was expressed as a percentage of injected dose per organ (see Supplementary Table 1). The gold content per gram of tissue was also estimated by neutron activation analysis (NAA) (see Supplementary Figure 1). These results confirm the expected accumulation of NSs within the liver and spleen and clearance from the tumor in both targeted and untargeted NS-treated animals at a late time point.

In addition to potential imaging applications of NSRGDfK, the feasibility of using these targeted probes for improved in vivo photothermal efficiency was also investigated. Assuming the targeted NSs were more biologically effective as thermal therapy conduits, we expected to observe more necrosis in tumors laden with these particles than the untargeted particles if a subablative thermal treatment was delivered. First, an in vivo model for subablative thermal treatment was created. This was based on an Arrhenius mathematical prediction model ${ }^{32}$ of thermal injury, which predicted that a sustained stable temperature of $47^{\circ} \mathrm{C}$ for 10 minutes would result in $\sim 50 \%$ cell death. Next, in a cohort of nude mice harboring $\sim 1 \mathrm{~cm}$ subcutaneous HCT116 colorectal cancers on their right thighs, laser settings that would result in these temperatures were established. The NSs were injected intravenously and subablative thermal treatments were conducted 24 hours after the injection. Finally, the effect of such treatment was compared between two mice injected
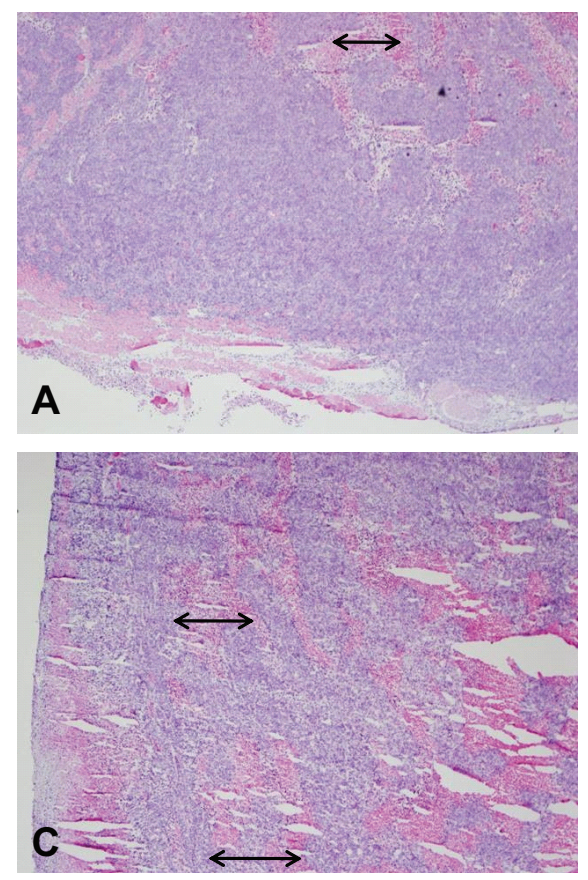

with similar particle concentrations ( $\sim 40 \mathrm{fmol})$ of untargeted and targeted NSs. The targeted NSs treatment group had more profound hemorrhage during treatment than the untargeted NSs treatment group. Greater amounts of hemorrhagic and necrotic debris were evident within the tumor core upon sectioning of the tumors from the targeted treatment group than the untargeted treatment group. As noted in Figure 5, the lower magnification images ( $\mathrm{A}$ and $\mathrm{C}, 4 \times$ ) demonstrate greater necrosis in the vascular-targeted group, suggestive of more significant vascular disruption. The results suggest that greater tumor and tumor vascular specificity via the active targeting technique improves the efficacy of thermal therapy. The observed enhancement in therapeutic efficacy is attributed to the higher tumor concentrations of targeted NSs as well as the possibly more intense focal temperature hot spots generated in the vicinity of vascular-targeted NSs, resulting in more vascular disruption.

\section{Conclusion}

In this work we investigated the binding affinity of cyclo(RGDfK) peptide and the antagonist IAC, as well as their NS conjugates to the integrin $\alpha_{\mathrm{v}} \beta_{3}$ by ELISA. We found that IAC has similar affinity to integrin $\alpha_{v} \beta_{3}$ as RGDfK in the competitive ELISA and the sandwich ELISA and that the difference is not as significant as previous reports. ${ }^{27}$ Because of the relatively small difference in the binding affinity to
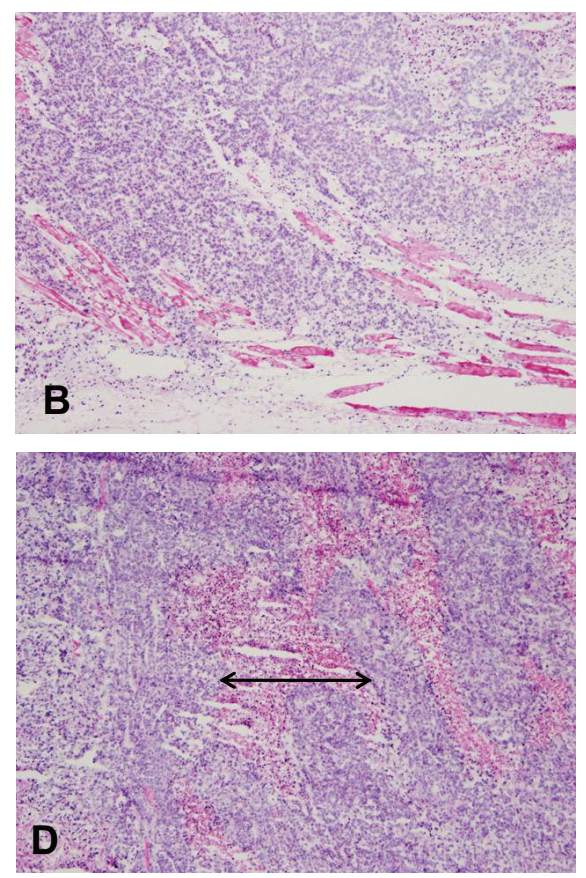

Figure $\mathbf{5}$ Hematoxylin and eosin stain of tumors excised immediately following sub-ablative thermal therapy using untargeted (A and B) NSs and vascular-targeted (C and $\mathbf{D})$ NSs. Images on the left are lower magnification images (A and C, $4 \times)$, whereas images on the right are higher magnification images (B and $\mathbf{D}$, I0 $)$. Arrows represent the width of areas of necrosis observed.

Abbreviation: NSs, gold nanoshells. 
integrin $\alpha_{\mathrm{v}} \beta_{3}$ between NS-RGDfK and NS-IAC, we pursued further experiments with NS-RGDfK. We confirmed potent, selective, and specific NS-RGDfK adhesion to integrin $\alpha_{\mathrm{v}} \beta_{3}$-expressing U87 tumor cells by silver staining. We also documented in vivo tumor-specific accumulation of conjugated NSs and nonspecific accumulation of probe within the liver and spleen at four different time points. Biodistribution analysis of untargeted and targeted NSs by measurement of residual radioactivity and NAA confirmed that both targeted and untargeted NSs are cleared from the circulation by the liver and spleen and suggested that integrin $\alpha_{\mathrm{v}} \beta_{3}$ targeting using RGDfK improved NS accumulation in tumors. Lastly, we confirmed increased biological efficacy of conjugated NSs for potential thermoablation applications.

As demonstrated in multiple cancers, integrin $\alpha_{v} \beta_{3}$ is a crucial cell adhesion and signaling molecule that promotes the proliferation, survival, drug-resistant properties, and metastatic potential of cancer cells. This specific and preferential overexpression noted across multiple tumor types makes targeting of integrin $\alpha_{\mathrm{v}} \beta_{3}$ for imaging and therapy particularly appealing, as it offers a class solution to the challenge posed by heterogeneity of tumor-specific antigens across diverse tumor types. Furthermore, integrin $\alpha_{v} \beta_{3}$ localization to angiogenic neovascular endothelia offers greater targeting potential for larger biomolecules and nanoparticles that are less capable of penetrating deep into tumor parenchyma across interstitial spaces. We demonstrate here that integrin $\alpha_{\mathrm{v}} \beta_{3}$-targeted NSs serve as potent theranostic agents that facilitate enhanced imaging and treatment of tumors.

\section{Acknowledgments}

This work was supported by NIST ATP Cooperative Agreement Number 70NANB4H3040 and NIH 1R21CA133691. We thank Pharmaceutical Research and Manufacturers of America Foundation for their support of Dr Xie's work. We thank Dr Narasimhan Danthi at Molecular Imaging Laboratory of National Institutes of Allergies and Infectious Diseases for kindly providing us with the IAC. We also thank Dr Ting Tung A Chang for his imaging technical support.

\section{Disclosure}

The authors report no conflicts of interest in this work.

\section{References}

1. James WD, Hirsch LR, West JL, O'Neal PD, Payne JD. Application of INAA to the build-up and clearance of gold nanoshells in clinical studies in mice. J Radioanal Nucl Chem. 2007;271(2):455-459.

2. Xie H, Wang ZJ, Bao A, Goins B, Phillips WT. In vivo PET imaging and biodistribution of radiolabeled gold nanoshells in rats with tumor xenografts. Int J Pharm. 2010;395:324-330.
3. O'Neal DP, Hirsch LR, Halas NJ, Payne JD, West JL. Photo-thermal tumor ablation in mice using near infrared-absorbing nanoparticles. Cancer Lett. 2004;209(2):171-176.

4. Hirsch LR, Stafford RJ, Bankson JA, et al. Nanoshell-mediated nearinfrared thermal therapy of tumors under magnetic resonance guidance. Proc Natl Acad Sci U S A. 2003;100(23):13549-13554.

5. Cai W, Shin DW, Chen K, et al. Peptide-labeled near-infrared quantum dots for imaging tumor vasculature in living subjects. Nano Lett. 2006; 6(4):669-676.

6. Huang YF, Chang HT, Tan W. Cancer cell targeting using multiple aptamers conjugated on nanorods. Anal Chem. 2008;80(3):567-572.

7. Diagaradjane P, Shetty A, Wang JC, et al. Modulation of in vivo tumor radiation response via gold nanoshell-mediated vascular-focused hyperthermia: characterizing an integrated antihypoxic and localized vascular disrupting targeting strategy. Nano Lett. 2008;8(5):1492-1500.

8. Ruoslahti E. RGD and other recognition sequences for integrins. Annu Rev Cell Dev Biol. 1996;12:697-715.

9. Hood JD, Cheresh DA. Role of integrins in cell invasion and migration. Nat Rev Cancer. 2002;2(2):91-100.

10. Xiong JP, Stehle T, Diefenbach B, et al. Crystal structure of the extracellular segment of integrin alpha Vbeta3. Science. 2001;294(5541): 339-345.

11. Cairns RA, Khokha R, Hill RP. Molecular mechanisms of tumor invasion and metastasis: an integrated view. Curr Mol Med. 2003;3(7): 659-671.

12. Felding-Habermann B. Integrin adhesion receptors in tumor metastasis. Clin Exp Metastasis. 2003;20(3):203-213.

13. Schmieder AH, Winter PM, Caruthers SD, et al. Molecular MR imaging of melanoma angiogenesis with alphanubeta3-targeted paramagnetic nanoparticles. Magn Reson Med. 2005;53(3):621-627.

14. Ellegala DB, Leong-Poi H, Carpenter JE, et al. Imaging tumor angiogenesis with contrast ultrasound and microbubbles targeted to alpha(v) beta3. Circulation. 2003;108(3):336-341.

15. Dayton PA, Pearson D, Clark J, et al. Ultrasonic analysis of peptide- and antibody-targeted microbubble contrast agents for molecular imaging of alphavbeta3-expressing cells. Mol Imaging. 2004;3(2):125-134.

16. Tkachenko AG, Xie H, Liu Y, et al. Cellular trajectories of peptidemodified gold particle complexes: comparison of nuclear localization signals and peptide transduction domains. Bioconjug Chem. 2004;15(3): 482-490.

17. Wang W, Ke S, Wu Q, et al. Near-infrared optical imaging of integrin alphavbeta3 in human tumor xenografts. Mol Imaging. 2004;3(4): 343-351.

18. Achilefu S, Bloch S, Markiewicz MA, et al. Synergistic effects of light-emitting probes and peptides for targeting and monitoring integrin expression. Proc Natl Acad Sci U S A. 2005;102(22):7976-7981.

19. Cheng Z, Wu Y, Xiong Z, Gambhir SS, Chen X. Near-infrared fluorescent RGD peptides for optical imaging of integrin alphavbeta3 expression in living mice. Bioconjug Chem. 2005;16(6):1433-1441.

20. Chen X, Conti PS, Moats RA. In vivo near-infrared fluorescence imaging of integrin alphavbeta 3 in brain tumor xenografts. Cancer Res. 2004;64(21):8009-8014.

21. Haubner R, Kuhnast B, Mang C, et al. [18F]Galacto-RGD: synthesis, radiolabeling, metabolic stability, and radiation dose estimates. Bioconjug Chem. 2004;15(1):61-69.

22. Janssen ML, Oyen WJ, Dijkgraaf I, et al. Tumor targeting with radiolabeled alpha(v)beta(3) integrin binding peptides in a nude mouse model. Cancer Res. 2002;62(21):6146-6151.

23. Sadeghi MM, Krassilnikova S, Zhang J, et al. Detection of injuryinduced vascular remodeling by targeting activated alphavbeta 3 integrin in vivo. Circulation. 2004;110(1):84-90.

24. Max R, Gerritsen RR, Nooijen PT, et al. Immunohistochemical analysis of integrin alpha vbeta3 expression on tumor-associated vessels of human carcinomas. Int J Cancer. 1997;71(3):320-324.

25. Cai W, Chen X. Anti-angiogenic cancer therapy based on integrin alphavbeta3 antagonism. Anticancer Agents Med Chem. 2006;6(5): 407-428. 
26. Friess H, Langrehr JM, Oettle H, et al. A randomized multi-center phase II trial of the angiogenesis inhibitor Cilengitide (EMD 121974) and gemcitabine compared with gemcitabine alone in advanced unresectable pancreatic cancer. BMC Cancer. 2006;6:285.

27. Burnett CA, Xie J, Quijano J, et al. Synthesis, in vitro, and in vivo characterization of an integrin alpha(v)beta(3)-targeted molecular probe for optical imaging of tumor. Bioorg Med Chem. 2005; 13(11):3763-3771.

28. Haubner R, Wester HJ, Burkhart F, et al. Glycosylated RGD-containing peptides: tracer for tumor targeting and angiogenesis imaging with improved biokinetics. J Nucl Med. 2001;42(2):326-336.

29. Hutchinson JH, Halczenko W, Brashear KM, et al. Nonpeptide alphavbeta3 antagonists. 8. In vitro and in vivo evaluation of a potent alphavbeta3 antagonist for the prevention and treatment of osteoporosis. J Med Chem. 2003;46(22):4790-4798.

30. Oldenburg SJ, Jackson JB, Westcott SL, Halas NJ. Infrared extinction properties of gold nanoshells. Appl Phys Lett. 1999;111:2897.

31. Bao A, Phillips WT, Goins B, et al. Setup and characterization of a human head and neck squamous cell carcinoma xenograft model in nude rats. Otolaryngol Head Neck Surg. 2006;135(6):853-857.
32. Welsch AJ, van Gemert MJC, editors. Optical-Thermal Response of Laser-Irradiated Tissue. New York: Plenum Press; 1995.

33. Ramos $\mathrm{OH}$, Kauskot A, Cominetti MR, et al. A novel alpha(v)beta (3)-blocking disintegrin containing the RGD motive, DisBa-01, inhibits bFGF-induced angiogenesis and melanoma metastasis. Clin Exp Metastasis. 2008;25(1):53-64.

34. Hu DD, Barbas CF, Smith JW. An allosteric Ca2+ binding site on the beta3-integrins that regulates the dissociation rate for RGD ligands. J Biol Chem. 1996;271(36):21745-21751.

35. Xie H, Wang Z, Bao A, Goins B, Phillips WT. Radiolabeled Gold Nanoshells for In Vivo Imaging: Example of Methodology for Initial Evaluation of Biodistribution of a Novel Nanoparticle NanoimagingBiomedical Nanotechnology: Pan Stanford Publishing Pte Ltd; in press 2010.

36. Xie H, Wang Z, Bao A, Goins B, Phillips WT, Coleman CL. Radiolabeling of Gold Nanoshells with Cu-64 and In-111 for PET and SPECT Imaging in Rats. Proceedings of the Nanotech Conference and Expo; 2009 May 3-7; Houston Texas; 2009. Vol 2:1-4 


\section{Supplementary data \\ Biodistribution studies}

All the animals were used in the imaging studies were anesthetized and then sacrificed at 46 hours postinjection. The organs of interest were removed and wet weighed. The radioactivity in the tissues was measured using a $\gamma$-counter (Wallac 1480, Perkin Elmer Life Sciences, Boston, MA, USA). The radioactivity of the tissue samples was calibrated against a known aliquot of the injectate. The percentage injected dose per organ (\%ID/organ) values were calculated using the following equation:

$$
\% \frac{\mathrm{ID}}{\text { Organ }}=\frac{(\mathrm{cpm} \text { in sample }- \text { background }) \times(\text { correction factor }) \times 100}{(\mathrm{cpm} \text { in standard }) \times \frac{(\text { injection volume })}{(\text { standard volume })}}
$$

Major organs that showed high concentrations of other types of nanoparticles in previous biodistribution studies ${ }^{1-4}$ were also subjected to neutron activation analysis (NAA). Portions of tumor, spleen, liver, lung, and kidney tissue

Table SI Biodistribution data of ${ }^{64} \mathrm{Cu}-\mathrm{NS}$ and ${ }^{64} \mathrm{Cu}-\mathrm{NS}-\mathrm{RGDfK}$ in HNSCC xenograft-bearing nude rats at 46 hours postinjection.

\begin{tabular}{lll}
\hline Organ & ${ }^{64} \mathbf{C u}-\mathbf{N S}$ & ${ }^{64} \mathbf{C u}-\mathbf{N S}-\mathbf{R G D F K}$ \\
\hline Tumor & 0.97 & 1.20 \\
Spleen & 4.93 & 8.77 \\
Liver & 16.39 & $13.0 \mathrm{I}$ \\
Lung & 0.31 & 0.34 \\
Kidney & 1.76 & 1.58 \\
Blood & 2.26 & 1.98 \\
Skin & 4.80 & 5.04 \\
Muscle & 6.80 & 7.47 \\
Bone & 6.02 & 8.97 \\
Heart & 0.17 & 0.20 \\
Stomach & 0.76 & 1.22 \\
Intestine & 5.21 & 7.83 \\
Cecum & 3.16 & 4.62 \\
Bladder & 0.02 & 0.11 \\
Testis & 0.62 & 0.61 \\
Brain & 0.06 & 0.06 \\
\hline
\end{tabular}

Note: Data are presented as percentage injected dose per organ (\% ID/organ).

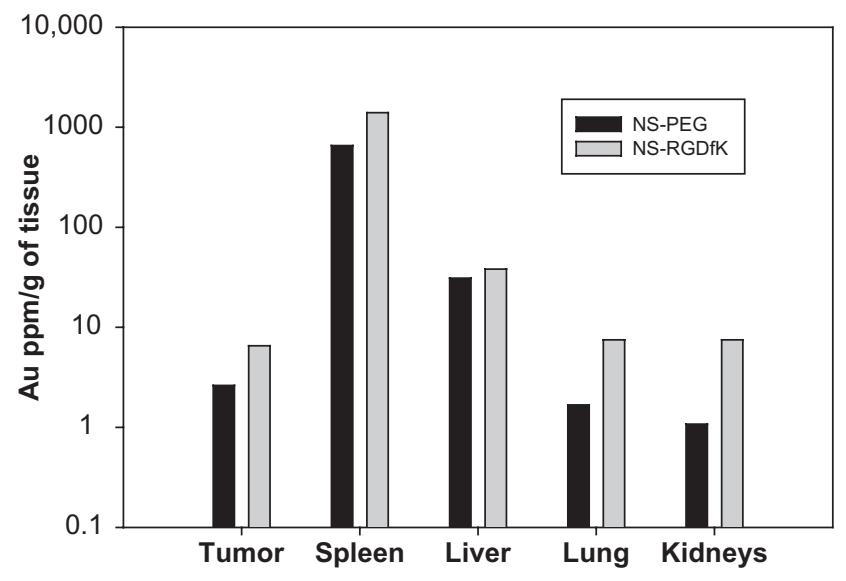

Figure SI Neutron activation analysis for concentration of gold in tumor, spleen, liver, lung, and kidney at 46 hours postinjection of NS-PEG (control) and NS-RGDfK.

were collected, wet weighed, and allowed to decay for 2 weeks. The samples were placed into precleaned and labeled polyethylene irradiation vials. After the wet sample weight was calculated and recorded, the samples were covered and dried under a heat lamp for 48 hours before delivery into the high temperature nuclear reactor core for NAA gold measurements. The procedure of NAA for trace gold quantification in animal tissues has been described elsewhere. ${ }^{5}$ The data were reported as gold mass versus the original wet sample mass.

\section{References}

1. Akiyama Y, Mori T, Katayama Y, Niidome T. The effects of PEG grafting level and injection dose on gold nanorod biodistribution in the tumor-bearing mice. J Control Release. 2009;139(1):81-84.

2. Cai W, Shin DW, Chen K, et al. Peptide-labeled near-infrared quantum dots for imaging tumor vasculature in living subjects. Nano Lett. 2006; 6(4):669-676.

3. Liu Z, Cai W, He L, et al. In vivo biodistribution and highly efficient tumour targeting of carbon nanotubes in mice. Nat Nanotechnol. 2007; 2(1):47-52.

4. Xie H, Wang ZJ, Bao A, Goins B, Phillips WT. In vivo PET imaging and biodistribution of radiolabeled gold nanoshells in rats with tumor xenografts. Int J Pharm. 2010;395:324-330.

5. James WD, Hirsch LR, West JL, O'Neal PD, Payne JD. Application of INAA to the build-up and clearance of gold nanoshells in clinical studies in mice. J Radioanal Nucl Chem. 2007;271(2):455-459.
International Journal of Nanomedicine

\section{Publish your work in this journal}

The International Journal of Nanomedicine is an international, peerreviewed journal focusing on the application of nanotechnology in diagnostics, therapeutics, and drug delivery systems throughout the biomedical field. This journal is indexed on PubMed Central,

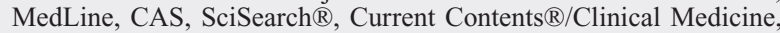

\section{Dovepress}

Journal Citation Reports/Science Edition, EMBase, Scopus and the Elsevier Bibliographic databases. The manuscript management system is completely online and includes a very quick and fair peer-review system, which is all easy to use. Visit http://www.dovepress.com/ testimonials.php to read real quotes from published authors. 